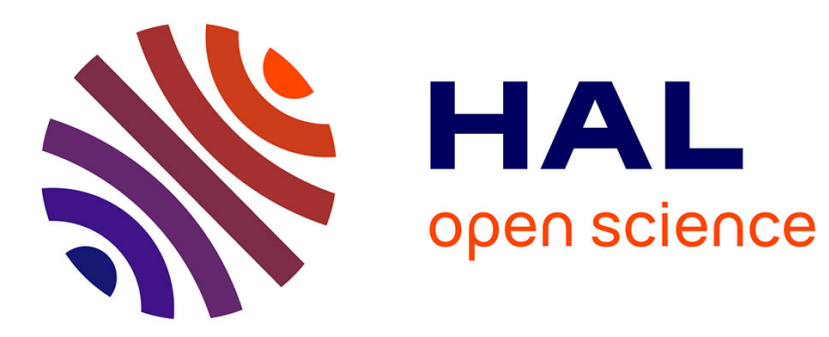

\title{
A lenticular version of a von Neumann inequality
}

Bernhard Beckermann, Michel Crouzeix

\section{To cite this version:}

Bernhard Beckermann, Michel Crouzeix. A lenticular version of a von Neumann inequality. Archiv der Mathematik, 2006, 86 (4), pp.352-355. 10.1007/s00013-005-1533-5 . hal-00447535

\section{HAL Id: hal-00447535 \\ https://hal.science/hal-00447535}

Submitted on 11 Dec 2017

HAL is a multi-disciplinary open access archive for the deposit and dissemination of scientific research documents, whether they are published or not. The documents may come from teaching and research institutions in France or abroad, or from public or private research centers.
L'archive ouverte pluridisciplinaire $\mathbf{H A L}$, est destinée au dépôt et à la diffusion de documents scientifiques de niveau recherche, publiés ou non, émanant des établissements d'enseignement et de recherche français ou étrangers, des laboratoires publics ou privés. 


\title{
A lenticular version of a von Neumann inequality
}

By

\author{
BERNHARD BECKERMANN and MiCHEL CROUZEIX
}

\begin{abstract}
We generalize to lens-shaped domains the classical von Neumann inequality for the disk.
\end{abstract}

1. Introduction. We will say that $L$ is a convex lens-shaped domain of the complex plane, with vertices $\sigma$ and $\sigma^{\prime}$, if

- either there exist two disks

$$
D_{1}:=\left\{z \in \mathbb{C} ;\left|z-\alpha_{1}\right|<r_{1}\right\} \text { and } D_{2}:=\left\{z \in \mathbb{C} ;\left|z-\alpha_{2}\right|<r_{2}\right\}
$$

such that $L=D_{1} \cap D_{2}, \sigma \neq \sigma^{\prime}$ and $\left\{\sigma, \sigma^{\prime}\right\}=\partial D_{1} \cap \partial D_{2}$,

- or there exist a disk and a half-plane

$$
D_{1}:=\left\{z \in \mathbb{C} ;\left|z-\alpha_{1}\right|<r_{1}\right\} \text { and } \Pi_{2}:=\left\{z \in \mathbb{C} ; \operatorname{Re} e^{i \theta}(z-\sigma)<0\right\}
$$

such that $L=D_{1} \cap \Pi_{2}, \sigma \neq \sigma^{\prime}$ and $\left\{\sigma, \sigma^{\prime}\right\}=\partial D_{1} \cap \partial \Pi_{2}$.

We will denote by $2 \alpha \in] 0, \pi]$ the angle of the lens $L$ at the vertices. We will consider also as a lens the limit case where $L=D_{1}=D_{2}$ is a disk. Then, any point of the boundary may be considered as a vertex and $\alpha=\frac{\pi}{2}$.

Now let us consider a bounded linear operator $A \in \mathcal{B}(H)$ on a complex Hilbert space $H$. We will say that the operator $A$ is of the lenticular $L$-type if we have

- $\left\|A-\alpha_{1} I\right\| \leqq r_{1}$ and $\left\|A-\alpha_{2} I\right\| \leqq r_{2}$, if $L=D_{1} \cap D_{2}$,

- $\left\|A-\alpha_{1} I\right\| \leqq r_{1}$ and $\operatorname{Re} e^{i \theta}((A-\sigma) v, v) \leqq 0, \forall v \in H$, if $L=D_{1} \cap \Pi_{2}$.

Mathematics Subject Classification (2000): 47A25 ; 47A30.

The first named author is partly supported by INTAS network NeCCA 03-51-6637, and in part by the Ministry of Science and Technology (MCYT) of Spain and the European Regional Development Fund (ERDF) through the grant BFM2001-3878-C02-02. 
In this paper, the norm used for a linear operator on a Hilbert space $H$ (as well as for a matrix) is always the operator norm induced by the hilbertian structure.

The aim of this paper is to prove the following result.

Theorem 1. Let $L$ be a convex lens-shaped domain of the complex plane with angle $2 \alpha$. There exists a best constant $C(\alpha) \in \mathbb{R}$ such that the inequality

$$
\|p(A)\| \leqq C(\alpha) \sup _{z \in L}|p(z)|,
$$

holds for all polynomials $p: \mathbb{C} \rightarrow \mathbb{C}$, for all linear operators $A \in \mathcal{B}(H)$ of L-type and for all Hilbert spaces $H$. Furthermore this constant, which is only depending on the angle $\alpha$, is a continuous decreasing function of $\alpha \in\left(0, \frac{\pi}{2}\right]$, and we have the estimate

$$
\frac{\pi}{2 \alpha} \sin \alpha \leqq C(\alpha) \leqq \min \left(2+2 / \sqrt{3}, \frac{\pi-\alpha}{\alpha}\right) .
$$

Note that for $\alpha=\frac{\pi}{2}$, which corresponds to the case where $L$ is a disk, we have $C\left(\frac{\pi}{2}\right)=1$, and we recover a famous von Neumann inequality [4]. Except for this value $\frac{\pi}{2}$, we do not know the exact values of $C(\alpha)$. A small improvement

$$
C(\alpha) \leqq \frac{\pi-\alpha}{\pi}\left(2-\frac{2}{\pi} \log \tan \left(\frac{\alpha \pi}{4(\pi-\alpha)}\right)\right)
$$

of the upper bound in (2) can be deduced from Theorem 4.2 in [1].

Theorem 1 can be generalized in several directions. For instance, by Mergelyan's Theorem, the inequality (1) remains valid if instead of polynomials we take $p$ holomorphic in $L$ and continuous in $\bar{L}$. The theorem is also valid in a completely bounded form. More precisely, if we consider now polynomial functions $P$ with matrix values: $\mathbb{C} \in z \mapsto P(z)=\left(p_{i j}(z)\right) \in \mathbb{C}^{n, n}$, then there exists a continuous decreasing function $C_{c b}(\alpha)$ (which satisfies the bounds given for $C(\alpha)$ ) such that the inequality

$$
\|P(A)\| \leqq C_{c b}(\alpha) \sup _{z \in L}\|P(z)\|
$$

holds for all polynomials $P$ with matrix values, for all linear operators $A \in \mathcal{B}(H)$ of type $L$ and for all Hilbert spaces $H$. The adverb completely points out the fact that the inequality holds independently of the size $n$ of the matrices. We do not know if $C(\alpha)=C_{c b}(\alpha)$ or not.

We should mention that a preliminary version of this theorem, in the particular case where $L$ has a straight face, has been implicitly used in [2] to study the convergence of the GMRES method.

2. The proof. Our proof of Theorem 1 is heavily based on the result of the paper [3], that we recall now. Let $S_{\alpha}$ be a convex sector of the complex plane with angle $2 \alpha$. An 
operator $B \in \mathcal{B}(H)$ is said $S_{\alpha}$-accretive iff $(B v, v) \in \overline{S_{\alpha}}$, for all $v \in H$ satisfying $\|v\|=1$. The result proved in [3] is

there exists a best constant $C_{\alpha} \in \mathbb{R}$ such that the inequality

$$
\|r(B)\| \leqq C_{\alpha} \sup _{z \in S_{\alpha}}|r(z)|,
$$

holds for all rational functions bounded in $S_{\alpha}$ and for all $S_{\alpha}$-accretive operators $B$. Furthermore this constant $C_{\alpha}$ (which only depends of $\alpha$ ) is a continuous and decreasing function of $\alpha$ and it satisfies the estimates

$$
\frac{\pi}{2 \alpha} \sin \alpha \leqq C_{\alpha} \leqq \min \left(2+2 / \sqrt{3}, \frac{\pi-\alpha}{\alpha}\right) .
$$

Therefore it is sufficient to prove that $C_{\alpha}=C(\alpha)$ for getting the theorem.

We turn now to the proof of this equality. Without loss of generality, we can assume that the vertices of $L$ are $\sigma=0$ and $\sigma^{\prime}=1$, and that $\operatorname{Im} \alpha_{1}<0$. We introduce the rational function $g(z):=\frac{z}{z-1}$. It is easily seen that $g$ is an involution and that $g$ realizes a bijection of the disk $D_{j}:=\left\{z \in \mathbb{C} ;\left|z-\alpha_{j}\right|<\left|\alpha_{j}\right|\right\}$ onto the half-plane $P_{j}:=\left\{z \in \mathbb{C} ; \operatorname{Re} \bar{\alpha}_{j} z<0\right\}$. In the case where the lens has a straight face $L=D_{1} \cap \Pi_{2}$ with $\Pi_{2}:=\{z \in \mathbb{C} ; \operatorname{Re} i z<0\}$, we remark also that $g$ realizes a bijection of the half-plane $\Pi_{2}$ onto the half-plane $P_{2}:=$ $\{z \in \mathbb{C} ; \operatorname{Re} i z>0\}$. Therefore $g$ is a bijection of the lens $L$ onto the sector $S_{\alpha}=P_{1} \cap P_{2}$. Note that the sector and the lens have the same angle $2 \alpha$ and that $1 \notin S_{\alpha}$.

Let us consider now a linear operator $A$ such that 1 does not belong to its spectrum $\sigma(A)$, and we set $B=g(A)=A(A-I)^{-1}$. It is easily seen that $(B-I)(A-I)=I$, thus $1 \notin \sigma(B)$, and $A=g(B)$.

Using that $\operatorname{Re} \alpha_{j}=\frac{1}{2}$, we remark by setting $v=(A-I) w$ that

$$
\begin{aligned}
& \left|\alpha_{j}\right|^{2}\|w\|^{2}-\left\|\left(A-\alpha_{j} I\right) w\right\|^{2} \geqq 0, \forall w \in H, \\
& \Longleftrightarrow\|A w\|^{2}-2 \operatorname{Re} \bar{\alpha}_{j}(A w, w) \leqq 0, \forall w \in H, \\
& \Longleftrightarrow 2 \operatorname{Re} \bar{\alpha}_{j}(A w,(A-I) w) \leqq 0, \forall w \in H, \\
& \Longleftrightarrow \operatorname{Re} \bar{\alpha}_{j}(B v, v) \leqq 0, \forall v \in H .
\end{aligned}
$$

In the case where $L$ has a straight face, we also remark that

$$
\begin{aligned}
& \operatorname{Im}(A w, w) \geqq 0, \forall w \in H, \\
& \Longleftrightarrow \operatorname{Im}(A w,(A-I) w) \leqq 0, \forall w \in H, \\
& \Longleftrightarrow \operatorname{Im}(B v, v) \leqq 0, \forall v \in H .
\end{aligned}
$$

Therefore if the linear operator $A$ is of $L$-type, then the operator $B$ is $S_{\alpha}$-accretive. Conversely if $B$ is $S_{\alpha}$-accretive then $1 \notin \sigma(B)$ (since $1 \notin S_{\alpha}$ ) and $A=g(B)$ is of $L$-type. 
Let us consider now a polynomial $p$ and set $r(z)=p(g(z))$, then we have $p(A)=r(B)$ and $\sup |r(z)|=\sup |p(\zeta)|$. We deduce from (3) that

$$
\begin{gathered}
z \in S_{\alpha} \quad \zeta \in L \\
\|p(A)\| \leqq C_{\alpha} \sup _{\zeta \in L}|p(\zeta)| .
\end{gathered}
$$

Note that, if $1 \in \sigma(A)$, then for $0<\varepsilon<1$, the operator $A_{\varepsilon}:=(1-\varepsilon) A$ is of $L$-type and $1 \notin \sigma\left(A_{\varepsilon}\right)$, which shows that the previous inequality is still valid by using a limit argument. Therefore we have $C(\alpha) \leqq C_{\alpha}$.

Conversely if we consider a rational function $r$ bounded in $S_{\alpha}, p(z)=r(g(z))$ is a rational function bounded in $L$. Note that $p$ is then a uniform limit in $L$ of a sequence of polynomial functions, therefore the estimate (1) is still valid. We deduce that

$$
\|r(B)\| \leqq C(\alpha) \sup _{z \in S_{\alpha}}|r(z)|,
$$

which implies $C(\alpha) \geqq C_{\alpha}$, and thus finally $C(\alpha)=C_{\alpha}$.

The proofs would be the same for the completely bounded form of our estimates.

\section{References}

[1] C. Badea, M. CrouzeiX and B. Delyon, Convex domains and $K$-spectral sets. Math. J. 252(2), 345-365 (2006).

[2] B. Beckermann, S. A. Goreinov and E. E. Tyrtyshnikov, Some remarks on the Elman estimate for GMRES. SIAM J. Matrix Anal. Appl., to appear.

[3] M. CrouzeiX AND B. Delyon, Some estimates for analytic functions of strip or sectorial operators. Arch. Math. 81, 553-566 (2003).

[4] J. von NeumanN, Eine Spektraltheorie für allgemeine Operatoren eines unitären Raumes. Math. Nachr. 4, 258-281 (1951).

\section{B. Beckermann}

Laboratoire de Mathématiques Paul Painlevé

UMR CNRS 8524

Bâtiment M2 - Cité Scientifique

Université des Sciences et Technologies de Lille

F-59655 Villeneuve d'Ascq cedex

France

bernhard.beckermann@univ-lille1.fr

\section{Crouzeix}

IRMAR

UMR CNRS 6625

Université de Rennes 1

Campus de Beaulieu

F-35042 Rennes cedex

France

michel.crouzeix@univ-rennes1.fr 